\title{
Parametric inference for stochastic differential equations with random effects in the drift coefficient
}

\author{
Alsukaini Mohammed Sari*, Wang Xiang-Jun \\ School of Mathematics and Statistics, Huazhong University of Science and Technology, Wuhan, Hubei, 430074, P.R. China \\ *Corresponding author E-mail: mohammedsari@yahoo.com
}

\begin{abstract}
In this paper we focus on estimating the parameters in the stochastic differential equations (SDE's) with drift coefficients depending linearly on a random variables $\emptyset_{i}$ and $\mu_{i}$. The distributions of the random effects $\emptyset_{i}$ and $\mu_{i}$ are depends on unknown parameters from the continuous observations of the independent processes $\left(X_{i}(t), t \in\left[0, T_{i}\right], i=1, \ldots, n\right)$. When $\mu$ is an unknown parameter or restrict positive constant also studied in this paper. We propose the Gaussian distribution for the random effect $\emptyset_{i}$ and the exponential distribution for the random effect $\mu_{i}$, we obtained an explicit formulas for the likelihood functions in each case and find the maximum likelihood estimators of the unknown parameters in the random effects and for the unknown parameter $\mu$. Consistency and asymptotic normality are studied just when $\emptyset_{i}$ is normal random effect and $\mu$ is constant.
\end{abstract}

Keywords: Stochastic Differential Equations; Maximum Likelihood Estimator; Linear Random Effects; Fisher Information Matrix; Asymptotic Normality; Consistency.

\section{Introduction}

Stochastic differential equations play an important role in modeling various phenomena arising in fields as diverse as finance, physics, chemistry, engineering, biology, neuroscience and others, (Allen (2007)[1], Hindriks (2011) [2] Musiela and Rutkowski (2005) [3], Gugushvili, S. and P. Spreij [4] and Wong and Hajek (1985) [5] ).

Parameters estimation in stochastic differential equations is a rapidly expanding area of research; ( Nielsen, Madsen and Young(2000) [6], Sørensen (2004) [7]). Statistical estimation of parameters in the diffusion processes has been studied for a long time; Feigin [8] provided a useful historical overview of the early studies and introduced a general asymptotic theory of maximum likelihood estimation for continuous diffusion processes. In the recent years, the stochastic differential equations with random effects have been considered in various works (Overgaard et al (2005) [9] Tornøe et al. (2005) [10] Ditlevsen and De Gaetano (2005)) and have been the subject of various applications such as pharmacokinetic/pharmacodynamics, neuronal modeling and modeling of electrical circuits (Delattre and Lavelle 2013 [11], Klim, Søren [12],Christoffer [10] ,Donnet and Samson 2013 [13], Picchini et al. 2010 [14], kampowsky and et al(1992)) [15].The problem of estimating parameters in SDE models is not straightforward, except in a simple cases. A natural approach would be likelihood inference, but the transition densities of the process are rarely known, and thus it is usually not possible to write the likelihood function explicitly. Many references proposed approximations for the unknown likelihood function, for general mixed SDEs an approximations of the likelihood have been proposed (Picchini and Ditlevsen, (2011) [16]), linearization (Beal and Sheiner (1982) [17])), approximate the transition density (Pedersen (1995) [18] Brandt and Santa-Clara (2002) [19] Nicolau (2002) [20], Hurn and Lindsay (1999) [21]), by solving numerically the
Kolmogorov partial differential equations satisfied by the transition density (Lo [22] (1988)) or approximating the conditional transition density of the diffusion process given the random effects by a Hermit expansion، (Aït-Sahalia [23] (2002)). Delattre [24] studied the maximum likelihood estimator for random effects in more generally for fixed $\mathrm{T}$ and $\mathrm{n}$ tending to infinity and found an explicit expression for likelihood function and exact likelihood estimator by investigate the linear random effect in the drift (multiple case) together with a specific distribution for the random effect.

In this paper we consider the stochastic differential equation with (i- two random effects, ii- random effect and unknown parameter ,iii- random effect and constant ) in drift coefficient and suppose that the diffusion coefficient without random effect. We study $n$ real valued stochastic processes $\left(X_{i}(t), t \in\left[0, T_{i}\right], i=\right.$ $1, \ldots, n)$, with dynamics ruled by the following SDEs:

$$
\begin{array}{r}
d X_{i}(t)=b\left(X_{i}(t), \emptyset_{i}, \mu_{i}\right) d t+\sigma\left(X_{i}(t)\right) d W_{i}(t), X_{i}(0)=x^{i} \\
i=1, \ldots, n,
\end{array}
$$

Where $\mathrm{W}_{1}, \ldots, \mathrm{W}_{\mathrm{n}}$ are $\mathrm{n}$ independent wiener processes. In the first case, $\emptyset_{1}, \ldots, \emptyset_{\mathrm{n}}$ and $\mu_{1}, \ldots, \mu_{\mathrm{n}}$ are $\mathrm{n}$ i. i. d. random variables taking values in ( $\mathbb{R}$ and $\mathbb{R}^{+}$) respectively, $\emptyset_{1}, \ldots, \emptyset_{\mathrm{n}}, \mu_{1}, \ldots, \mu_{\mathrm{n}}$ and $\mathrm{W}_{1}, \ldots, \mathrm{W}_{\mathrm{n}}$ are independent and $\mathrm{x}^{\mathrm{i}}, \mathrm{i}=1, \ldots, \mathrm{n}$ are known real values. In The second case we suppose $\mu_{\mathrm{i}}=\mu$ as an unknown parameter in $\mathbb{R}$ and the third case we suppose $\mu$ as a positive constant. The functions $\mathrm{b}(\mathrm{x})$ and $\sigma(\mathrm{x})$ are known real valued functions. Each process $X_{i}(t)$ represents an individual, the variables $\emptyset_{i}$ and $\mu_{\mathrm{i}}$ represents the random effects of individual $\mathrm{i}$, the random variables $\emptyset_{1}, \ldots, \emptyset_{\mathrm{n}}$ have a common distribution $g(\varphi, \theta) \mathrm{d} v(\varphi)$ on $\mathbb{R}$ and the random variables $\mu_{1}, \ldots, \mu_{n}$ have a common distribution $\mathrm{h}(\mu, \beta) \mathrm{du}(\mu)$ on $\mathbb{R}^{+}$where $\theta$ and $\beta$ are an unknown parameters belonging to a set $\Theta \subset \mathbb{R}^{p}$ where $v$ and $\mathrm{u}$ are a dominating measures. 
Our goal is to estimate $\psi=(\theta, \beta)$ from the continuous observations $\left(X_{i}(t), t \in\left[0, T_{i}\right], i=1, \ldots, n\right)$ and estimate the unknown parameter $\mu$.We focus on a special case of linear random effect in the drift coefficient in the model (1), i.e. $b\left(x, \emptyset_{i}, \mu_{i}\right)=$ $\emptyset_{\mathrm{i}} \mathrm{b}\left(\mathrm{X}_{\mathrm{i}}(\mathrm{t})\right)+\mu_{\mathrm{i}}$, where $\mathrm{b}$ is a known real function and $\emptyset_{\mathrm{i}}$ is a Gaussian and $\mu_{\mathrm{i}}$ is an exponential in the first case,$\mu$ is unknown parameter in the second case and $\mu$ is constant in the third case. An explicit likelihood formula and the maximum likelihood estimators are obtained in the three cases; asymptotic properties are obtained in the third case only.

The structure of the paper is as follows. Section 2 contains the notation and assumptions. The general results of the estimation of the parameters are introduced in section 3 . In section 4 we study the asymptotic properties (consistency and asymptotic normality) of the estimators. Conclusion is given in section 5 .

\section{Notations and assumptions}

Consider $n$ real valued stochastic processes $\left(X_{i}(t), t \geq 0\right), i=$ $1, \ldots, \mathrm{n}$ with dynamics ruled by (1). The processes $\mathrm{W}_{1}, \ldots, \mathrm{W}_{\mathrm{n}}$ and the random variables $\emptyset_{1}, \ldots, \emptyset_{\mathrm{n}}$ and $\mu_{1}, \ldots, \mu_{\mathrm{n}}$ are defined on a common probability space $(\Omega, \mathcal{F}, \mathbb{P})$. Consider the filtration $\left(\mathcal{F}_{\mathrm{t}}, \mathrm{t} \geq 0\right)$ defined by $\mathcal{F}_{\mathrm{t}}=\sigma\left(\emptyset_{\mathrm{i}}, \mu_{\mathrm{i}}, \mathrm{W}_{\mathrm{i}}(\mathrm{s}), \mathrm{s} \leq \mathrm{t}, \mathrm{i}=1, \ldots, \mathrm{n}\right)$. As $\quad \mathcal{F}_{\mathrm{t}}=\sigma\left(\emptyset_{\mathrm{i}}, \mu_{\mathrm{i}}, \mathrm{W}_{\mathrm{i}}(\mathrm{s}), \mathrm{s} \leq \mathrm{t}\right) \mathrm{V} \mathcal{F}_{\mathrm{t}}^{\mathrm{i}}, \quad$ with $\quad \mathcal{F}_{\mathrm{t}}^{\mathrm{i}}=$ $\sigma\left(\emptyset_{\mathrm{i}}, \emptyset_{\mathrm{j}}, \mu_{\mathrm{i}}, \mu_{\mathrm{j}}, \mathrm{W}_{\mathrm{j}}(\mathrm{s}), \mathrm{s} \leq \mathrm{t}, \mathrm{j} \neq \mathrm{i}\right)$ independent of $\mathrm{W}_{\mathrm{i}}$, each process $\mathrm{W}_{\mathrm{i}}$ is a $\left(\mathcal{F}_{\mathrm{t}}, \mathrm{t} \geq 0\right)$-Brownian motion. Moreover, the random variables $\emptyset_{i}, \mu_{\mathrm{i}}$ are $\mathcal{F}_{0}$ - measurable. We assume that:

H1

i) The function $b(x, \varphi, \mu)$ is $C^{1}$ on $\mathbb{R} \times \mathbb{R}^{d} \times \mathbb{R}^{+}$and such that:

$\exists \mathrm{K}>0, \forall \mathrm{x} \in \mathbb{R}, \mathrm{b}^{2}(\mathrm{x}, \varphi, \mu) \leq \mathrm{K}\left(1+\mathrm{x}^{2}+|\varphi|^{2}+|\mu|^{2}\right)$,

ii) The function $\sigma(\mathrm{x})$ is $\mathrm{C}^{1}$ on $\mathbb{R}$ and

$\forall \mathrm{x} \in \mathbb{R}, \sigma^{2}(\mathrm{x}) \leq \mathrm{K}\left(1+\mathrm{x}^{2}\right)$.

From $\mathrm{H} 1$, the process $\left(\mathrm{X}_{\mathrm{i}}(\mathrm{t})\right)$ is well define and $\left(\emptyset_{\mathrm{i}}, \mu_{\mathrm{i}}, \mathrm{X}_{\mathrm{i}}(\mathrm{t})\right)$ adapted to filtration $\left(\mathcal{F}_{\mathrm{t}}, \mathrm{t} \geq 0\right)$.

The $\mathrm{n}$ processes $\left(\emptyset_{\mathrm{i}}, \mu_{\mathrm{i}}, \mathrm{X}_{\mathrm{i}}(\mathrm{t}), \mathrm{i}=1, \ldots, \mathrm{n}\right)$ are independent. For all $\varphi, \mu$ and all $x^{i} \in \mathbb{R}$, the stochastic differential equation

$d X_{i}^{\varphi, \mu}(t)=b\left(X_{i}^{\varphi, \mu}(t), \varphi, \mu\right) d t+\sigma\left(X_{i}^{\varphi, \mu}(t)\right) d W_{i}(t)$,

$X_{i}^{\varphi, \mu}(0)=x^{i}$

Admits a unique strong solution process $\left(X_{i}^{\varphi, \mu}(t), t \geq 0\right)$ adapted to filtration $\left(\mathcal{F}_{t}, t \geq 0\right)$. We deduce that the conditional distribution of $X_{i}$ given $\emptyset_{i}=\varphi$ and $\mu_{i}=\mu$ identical to the distribution $o$ $f X_{i}^{\varphi, \mu}$.

\section{A general results of parameters estimation}

\subsection{When $\varphi$ and $\mu$ are random effects}

\subsubsection{Exact likelihood}

We introduce the distribution $\mathrm{Q}_{\varphi, \mu}^{\mathrm{x}^{\mathrm{i}}, \mathrm{T}_{\mathrm{i}}}$ of $\left(\mathrm{X}_{\mathrm{i}}^{\varphi, \mu}(\mathrm{t}), \mathrm{t} \in\left[0, \mathrm{~T}_{\mathrm{i}}\right]\right)$.

Let $P_{\psi}^{i}=g(\varphi, \theta) d v(\varphi) \otimes h(\mu, \beta) d u(\mu) \otimes Q_{\varphi}^{x^{i}, T_{i}}$ denote the joint distribution of $\left(\emptyset_{\mathrm{i}}, \mu_{\mathrm{i}}, \mathrm{X}_{\mathrm{i}}(\mathrm{t})\right)$ and let $\mathrm{Q}_{\psi}^{\mathrm{i}}$ denote the marginal distribution of $\left(\mathrm{X}_{\mathrm{i}}(\mathrm{t}), \mathrm{t} \in\left[0, \mathrm{~T}_{\mathrm{i}}\right]\right)$. Let us consider the following assumption:

$\mathrm{H} 2$ For $\mathrm{i}=1, \ldots, \mathrm{n}$ and for all $\varphi, \mu, \varphi^{\prime}, \mu^{\prime}$,
$\mathrm{Q}_{\varphi, \mu}^{\mathrm{x}^{\mathrm{i}}, \mathrm{T}_{\mathrm{i}}}\left(\int_{0}^{\mathrm{T}_{\mathrm{i}}} \frac{\mathrm{b}^{2}\left(\mathrm{x}_{\mathrm{i}}^{\varphi, \mu}(\mathrm{t}), \varphi^{\prime}, \mu^{\prime}\right)}{\sigma^{2}\left(\mathrm{x}_{\mathrm{i}}^{\varphi, \mu}(\mathrm{t})\right)} \mathrm{dt}<+\infty\right)=1$

Under H1-H2, the derivative of the distribution $Q_{\varphi, \mu}^{x^{i}, T_{i}}$ with respect to derivative of $Q^{\mathrm{i}}=Q_{\varphi_{0}, \mu_{0}}^{\mathrm{x}^{\mathrm{i}}, \mathrm{T}_{\mathrm{i}}}$ has the density:

$\frac{\mathrm{dQ}_{\varphi, \mu}^{\mathrm{x}^{\mathrm{i}}, \mathrm{T}_{\mathrm{i}}}}{\mathrm{dQ} Q^{\mathrm{i}}}\left(\mathrm{X}_{\mathrm{i}}\right)=\mathrm{L}_{\mathrm{T}_{\mathrm{i}}}\left(\mathrm{X}_{\mathrm{i}}, \varphi, \mu\right)$

$=\exp \left(\int_{0}^{\mathrm{T}_{\mathrm{i}}} \frac{\mathrm{b}\left(\mathrm{X}_{\mathrm{i}}(\mathrm{s}), \varphi, \mu\right)}{\sigma^{2}\left(\mathrm{X}_{\mathrm{i}}(\mathrm{s})\right)} \mathrm{dX} \mathrm{X}_{\mathrm{i}}(\mathrm{s})-\frac{1}{2} \int_{0}^{\mathrm{T}_{\mathrm{i}}} \frac{\mathrm{b}^{2}\left(\mathrm{X}_{\mathrm{i}}(\mathrm{s}), \varphi, \mu\right)}{\sigma^{2}\left(\mathrm{X}_{\mathrm{i}}(\mathrm{s})\right)} \mathrm{ds}\right)$

(for more details see Liptser and Shiryaev [25]).

The density is depending on the statistics:

$$
\begin{aligned}
& \mathrm{A}_{\mathrm{i}}=\int_{0}^{\mathrm{T}_{\mathrm{i}}} \frac{\mathrm{b}\left(\mathrm{X}_{\mathrm{i}}(\mathrm{s})\right)}{\sigma^{2}\left(\mathrm{X}_{\mathrm{i}}(\mathrm{s})\right)} \mathrm{d} \mathrm{X}_{\mathrm{i}}(\mathrm{s}), \mathrm{B}_{\mathrm{i}}=\int_{0}^{\mathrm{T}_{\mathrm{i}}} \frac{\mathrm{b}^{2}\left(\mathrm{X}_{\mathrm{i}}(\mathrm{s})\right)}{\sigma^{2}\left(\mathrm{X}_{\mathrm{i}}(\mathrm{s})\right)} \mathrm{ds}, \\
& \mathrm{C}_{\mathrm{i}}=\int_{0}^{\mathrm{T}_{\mathrm{i}}} \frac{1}{\sigma^{2}\left(\mathrm{X}_{\mathrm{i}}(\mathrm{s})\right)} \mathrm{d} \mathrm{X}_{\mathrm{i}}(\mathrm{s}), \mathrm{D}_{\mathrm{i}}=\int_{0}^{\mathrm{T}_{\mathrm{i}}} \frac{1}{\sigma^{2}\left(\mathrm{X}_{\mathrm{i}}(\mathrm{s})\right)} \mathrm{ds}, \\
& E_{i}=\int_{0}^{T_{i}} \frac{b\left(X_{i}(s)\right)}{\sigma^{2}\left(X_{i}(s)\right)} d s .
\end{aligned}
$$

By independent of individuals, $P_{\psi}=\bigotimes_{i=1}^{n} P_{\psi}^{i}$ is the distribution of $\left(\emptyset_{i}, \mu_{i}, X_{i}().\right), i=1, \ldots, n$ and $Q_{\psi}=\bigotimes_{i=1}^{n} Q_{\psi}^{i}$ is the distribution of the sample $\left(X_{i}(t), t \in\left[0, T_{i}\right], i=1, \ldots, n\right)$.

We can compute the density of $Q_{\psi}$ w.r.t. $Q=\bigotimes_{i=1}^{n} Q^{i}$ as follow:

$$
\begin{aligned}
& \gamma_{i}\left(X_{i}, \psi\right)=\frac{d Q_{\psi}^{i}}{d Q^{i}}\left(X_{i}\right) \\
& =\int_{\mathbb{R}^{+}} \int_{\mathbb{R}} L_{T_{i}}\left(X_{i}, \varphi, \mu\right) g(\varphi, \theta) h(\mu, \beta) d v(\varphi) d u(\mu)
\end{aligned}
$$

And the exact likelihood of whole sample $\left(X_{i}(t), t \in\left[0, T_{i}\right], i=\right.$ $1, \ldots, n)$ is

$\xi_{n}(\psi)=\prod_{i=1}^{n} \gamma_{i}\left(X_{i}, \psi\right)$

\subsubsection{The distributions of the random effects}

Consider model (1) with linear random effects in the drift coefficient $b(x, \varphi, \mu)=\varphi b(x)+\mu \quad$ where $\varphi \in \mathbb{R}, \mu \in \mathbb{R}^{+} \quad$ and $b(),. \sigma($.$) are known functions. We assume that:$

$\int_{0}^{T_{i}} \frac{b^{2}\left(X_{i}(s)\right)}{\sigma^{2}\left(X_{i}(s)\right)} d s<\infty, Q_{\varphi, \mu}^{x^{i}, T_{i}}-a . s$,

for all $\varphi, \mu$ and for $i=1, \ldots, n ; T_{i}=T, x^{i}=x$, so that $\left(X_{i}(t), t \in\right.$ $[0, T], i=1, \ldots, n)$ are $i . i . d$. We will use the well define statistics as follow:

$$
\begin{aligned}
A_{i} & =\int_{0}^{T} \frac{b\left(X_{i}(s)\right)}{\sigma^{2}\left(X_{i}(s)\right)} d X_{i}(s), B_{i}=\int_{0}^{T} \frac{b^{2}\left(X_{i}(s)\right)}{\sigma^{2}\left(X_{i}(s)\right)} d s \\
C_{i} & =\int_{0}^{T} \frac{1}{\sigma^{2}\left(X_{i}(s)\right)} d X_{i}(s), D_{i}=\int_{0}^{T} \frac{1}{\sigma^{2}\left(X_{i}(s)\right)} d s, \\
E_{i} & =\int_{0}^{T} \frac{b\left(X_{i}(s)\right)}{\sigma^{2}\left(X_{i}(s)\right)} d s
\end{aligned}
$$

So that the density $\gamma_{i}\left(X_{i}, \psi\right)$ is given by:

$$
\begin{aligned}
& \gamma_{i}\left(X_{i}, \psi\right)==\int_{\mathbb{R}^{+}} \int_{\mathbb{R}} \exp \left(\varphi A_{i}-\frac{1}{2} \varphi^{2} B_{i}\right) \exp \left(\left(\mu C_{i}-\right.\right. \\
& \left.\left.\frac{1}{2} \mu^{2} D_{i}\right)\right) \exp \left(-\varphi \mu E_{i}\right) g(\varphi, \theta) h(\mu, \beta) d v(\varphi) d u(\mu)
\end{aligned}
$$


For a general distributions, $g(\varphi, \theta) d v(\varphi)$ for the random effect $\emptyset_{i}$ and $h(\mu, \beta) d u(\mu)$ for the random effect $\mu$, it is not possible find an explicit expression for $\gamma_{i}\left(X_{i}, \psi\right)$ above, therefor we propose a specific distributions, Gaussian $\left(\lambda, \omega^{2}\right)$ for the random effect $\varphi$ and an exponential $(\beta)$ for the random effect, which will give an explicit likelihood and then find the maximum likelihood estimators of the unknown parameters. In the next proposition an evident expression for $\gamma_{i}\left(X_{i}, \psi\right)$ is obtained when the above distributions of the random effects is with unknown parameter $\psi=$ $\left(\lambda, \omega^{2}, \beta\right) \in \mathbb{R} \times \mathbb{R}^{+} \times \mathbb{R}^{+}$. The true value is denoted by $\psi_{0}=$ $\left(\lambda_{0}, \omega_{0}^{2}, \beta_{0}\right)$.

Proposition3.1 suppose that $g(\varphi, \theta) d v(\varphi)=\mathcal{N}\left(\lambda, \omega^{2}\right)$, and $h(\mu, \beta) d u(\mu)=\exp (\beta)$ then:

$\gamma_{i}\left(X_{i}, \psi\right)=\frac{\beta \sqrt{2 \pi}}{\sqrt{D_{i}\left(1+\omega^{2} B_{i}\right)-\omega^{2} E_{i}{ }^{2}}} \exp \left[\frac{\omega^{2} A_{i}{ }^{2}+2 \lambda A_{i}-\lambda^{2} B_{i}}{2\left(1+\omega^{2} B_{i}\right)}\right] \times$

$\exp \left[\frac{\left[\left(1+\omega^{2} B_{i}\right)\left(C_{i}-\beta\right)-A_{i} E_{i} \omega^{2}-\lambda E_{i}\right]^{2}}{2\left(1+\omega^{2} B_{i}\right)\left[D_{i}\left(1+\omega^{2} B_{i}\right)-\omega^{2} E_{i}^{2}\right]}\right]$.

Proof: From (5) we compute the joint density of $\left(\emptyset_{i}, \mu_{i}, X_{i}\right)$ :

$=$

$\int_{\mathbb{R}^{+}} \int_{\mathbb{R}} \exp \left(\varphi A_{i}-\frac{1}{2} \varphi^{2} B_{i}\right) \exp \left(\left(\mu C_{i}-\right.\right.$

$\left.\left.\frac{1}{2} \mu^{2} D_{i}\right)\right) \exp \left(-\varphi \mu E_{i}\right) \frac{1}{\sqrt{2 \pi \omega^{2}}} \exp \left(-\frac{1}{2 \omega^{2}}(\varphi-\right.$

$\left.\lambda)^{2}\right) \beta \exp (-\beta \mu) d v(\varphi) d u(\mu)$

We progress the first part of the exponent as follow:

$\left(\varphi A_{i}-\frac{1}{2} \varphi^{2} B_{i}\right)-\frac{1}{2 \omega^{2}}(\varphi-\lambda)^{2}-\varphi \mu E_{i}$

$=\varphi A_{i}-\frac{1}{2} \varphi^{2} B_{i}-\frac{1}{2 \omega^{2}} \varphi^{2}+\frac{1}{\omega^{2}} \varphi \lambda-\frac{1}{2 \omega^{2}} \lambda^{2}-\varphi \mu E_{i}$

$=\left(-\frac{1}{2} B_{i}-\frac{1}{2 \omega^{2}}\right) \varphi^{2}+\left(A_{i}-\mu E_{i}+\frac{1}{\omega^{2}} \lambda\right) \varphi-\frac{1}{2 \omega^{2}} \lambda^{2}$

$=-\frac{1}{2}\left[\left(B_{i}+\omega^{-2}\right)\left(\varphi^{2}-2 \frac{A_{i}-\mu E_{i}+\omega^{-2} \lambda}{B_{i}+\omega^{-2}} \varphi\right)-\frac{1}{2} \omega^{-2} \lambda^{2}\right]$

$=-\frac{1}{2\left(B_{i}+\omega^{-2}\right)^{-1}}\left(\varphi-\frac{A_{i}-\mu E_{i}+\omega^{-2} \lambda}{B_{i}+\omega^{-2}}\right)^{2}$

$+\frac{\left(A_{i}-\mu E_{i}+\omega^{-2} \lambda\right)^{2}}{2\left(B_{i}+\omega^{-2}\right)}-\frac{1}{2} \omega^{-2} \lambda^{2}$

By rearrange the first integral, the first part is normal depend on the random effect $\varphi$ with mean,

$m_{i}=\frac{\lambda+\omega^{2}\left(A_{i}-E_{i}\right)}{1+\omega^{2} B_{i}}$,

And variance,

$\sigma_{i}^{2}=\frac{\omega^{2}}{1+\omega^{2} B_{i}}$

By substituting in (5), the result of the first integral is:

$\exp \left[\frac{\left(A_{i}-\mu E_{i}+\omega^{-2} \lambda\right)^{2}}{2\left(B_{i}+\omega^{-2}\right)}-\frac{1}{2} \omega^{-2} \lambda^{2}\right] \times \frac{\beta}{\sqrt{1+\omega^{2} B_{i}}}$

Using this result in the second integral and developing the exponent as follow:

$$
\begin{aligned}
& \frac{\left(A_{i}-\mu E_{i}+\omega^{-2} \lambda\right)^{2}}{2\left(B_{i}+\omega^{-2}\right)}-\frac{1}{2} \omega^{-2} \lambda^{2}-\beta \mu+\mu C_{i}-\frac{1}{2} \mu^{2} D_{i} \\
& =\left(-\frac{1}{2} D_{i}+\frac{1}{2} \frac{E_{i}^{2} \omega^{2}}{1+\omega^{2} B_{i}}\right) \mu^{2}
\end{aligned}
$$

$+\left(C_{i}-\beta-\frac{2 A_{i} E_{i} \omega^{2}+2 \lambda E_{i}}{2\left(1+\omega^{2} B_{i}\right)}\right) \mu+\frac{A_{i}{ }^{2} \omega^{2}+2 \lambda A_{i}-B_{i} \lambda^{2}}{2\left(1+\omega^{2} B_{i}\right)}$

$=\frac{-1}{2 \alpha_{i}^{2}}\left(\mu-\varrho_{i}\right)^{2}+\frac{\varrho_{i}^{2}}{2 \alpha_{i}^{2}}+\frac{A_{i}{ }^{2} \omega^{2}+2 \lambda A_{i}-B_{i} \lambda^{2}}{2\left(1+\omega^{2} B_{i}\right)}$

Where,

$\varrho_{i}=\frac{\left(1+\omega^{2} B_{i}\right)\left(C_{i}-\beta\right)-A_{i} E_{i} \omega^{2}-\lambda E_{i}}{D_{i}\left(1+\omega^{2} B_{i}\right)-E_{i}^{2} \omega^{2}}$,

And

$\alpha_{i}^{2}=\left(\frac{\left(1+\omega^{2} B_{i}\right) D_{i}-E_{i}^{2} \omega^{2}}{1+\omega^{2} B_{i}}\right)^{-1}$.

Now, by splitting the result into two parts that are independent and dependent on the random effect $\mu$ respectively.

Then the second integral gives us $\gamma_{i}\left(X_{i}, \psi\right)$.

\subsubsection{Estimators of parameters of the random effects}

We use the maximum likelihood approach to estimate $\psi=$ $\left(\lambda, \omega^{2}, \beta\right)$, the likelihood function is written as:

$\xi_{n}(\psi)=\prod_{i=1}^{n} \gamma_{i}\left(X_{i}, \psi\right)$.

The logarithm of likelihood function is,

$\mathcal{L}_{n}(\psi)=\log \xi_{n}(\psi)$

$=\log (2 \pi)^{\frac{n}{2}}+\log \beta^{n}-\sum_{i=1}^{n} \log \left(D_{i}\left(1+\omega^{2} B_{i}\right)-E_{i}^{2} \omega^{2}\right)^{\frac{1}{2}}$

$+\sum_{i=1}^{n} \frac{A_{i}^{2} \omega^{2}+2 \lambda A_{i}-B_{i} \lambda^{2}}{2\left(1+\omega^{2} B_{i}\right)}$

$+\sum_{i=1}^{n} \frac{1}{2} \frac{\left(\left(1+\omega^{2} B_{i}\right)\left(C_{i}-\beta\right)-A_{i} E_{i} \omega^{2}-\lambda E_{i}\right)^{2}}{\left(1+\omega^{2} B_{i}\right)\left(D_{i}\left(1+\omega^{2} B_{i}\right)-E_{i}{ }^{2} \omega^{2}\right)}$

With score function

$S_{n}(\psi)=\left(\frac{\partial}{\partial \lambda} \mathcal{L}_{n}(\psi) \frac{\partial}{\partial \omega^{2}} \mathcal{L}_{n}(\psi) \frac{\partial}{\partial \beta} \mathcal{L}_{n}(\psi)\right)^{\prime}$

Where

$\frac{\partial}{\partial \lambda} \mathcal{L}_{n}(\psi)=\sum_{i=1}^{n} \frac{2 A_{i}-2 \lambda B_{i}}{2\left(1+\omega^{2} B_{i}\right)}$

$-\sum_{i=1}^{n} \frac{\left(\left(1+\omega^{2} B_{i}\right)\left(C_{i}-\beta\right)-A_{i} E_{i} \omega^{2}-\lambda E_{i}\right) \times E_{i}}{\left(1+\omega^{2} B_{i}\right)\left(D_{i}\left(1+\omega^{2} B_{i}\right)-E_{i}^{2} \omega^{2}\right)}$

$=\sum_{i=1}^{n} \frac{A_{i}}{1+\omega^{2} B_{i}}-\lambda \sum_{i=1}^{n} \frac{B_{i}}{1+\omega^{2} B_{i}}$

$+\lambda \sum_{i=1}^{n} \frac{E_{i}^{2}}{\left(1+\omega^{2} B_{i}\right)\left(D_{i}\left(1+\omega^{2} B_{i}\right)-E_{i}^{2} \omega^{2}\right)}$

$-\sum_{i=1}^{n} \frac{E_{i}\left(1+\omega^{2} B_{i}\right)\left(C_{i}-\beta\right)}{\left(1+\omega^{2} B_{i}\right)\left(D_{i}\left(1+\omega^{2} B_{i}\right)-E_{i}^{2} \omega^{2}\right)}$

$+\sum_{i=1}^{n} \frac{A_{i} E_{i}^{2} \omega^{2}}{\left(1+\omega^{2} B_{i}\right)\left(D_{i}\left(1+\omega^{2} B_{i}\right)-E_{i}^{2} \omega^{2}\right)}$

$=\lambda\left(\sum_{i=1}^{n} \frac{E_{i}^{2}}{\left(1+\omega^{2} B_{i}\right)\left(D_{i}\left(1+\omega^{2} B_{i}\right)-E_{i}^{2} \omega^{2}\right)}-\frac{B_{i}}{1+\omega^{2} B_{i}}\right)$

$+\sum_{i=1}^{n}\left[\frac{A_{i}}{1+\omega^{2} B_{i}}+\frac{A_{i} E_{i}^{2} \omega^{2}}{\left(1+\omega^{2} B_{i}\right)\left(D_{i}\left(1+\omega^{2} B_{i}\right)-E_{i}^{2} \omega^{2}\right)}-\frac{E_{i}\left(C_{i}-\beta\right)}{\left(D_{i}\left(1+\omega^{2} B_{i}\right)-E_{i}^{2} \omega^{2}\right)}\right]$.

$\frac{\partial}{\partial \beta} \mathcal{L}_{n}(\psi)=$ 
$\frac{n}{\beta}-\sum_{i=1}^{n} \frac{\left(\left(1+\omega^{2} B_{i}\right)\left(C_{i}-\beta\right)-A_{i} E_{i} \omega^{2}-\lambda E_{i}\right)\left(1+\omega^{2} B_{i}\right)}{\left(1+\omega^{2} B_{i}\right)\left(D_{i}\left(1+\omega^{2} B_{i}\right)-E_{i}^{2} \omega^{2}\right)}$

$=\frac{n}{\beta}-\sum_{i=1}^{n} \frac{\left(1+\omega^{2} B_{i}\right)\left(C_{i}-\beta\right)}{D_{i}\left(1+\omega^{2} B_{i}\right)-E_{i}^{2} \omega^{2}}$

$+\sum_{i=1}^{n} \frac{E_{i}\left(A_{i} \omega^{2}-\lambda\right)}{D_{i}\left(1+\omega^{2} B_{i}\right)-E_{i}^{2} \omega^{2}}$

$=\sum_{i=1}^{n} \frac{\left(1+\omega^{2} B_{i}\right)}{D_{i}\left(1+\omega^{2} B_{i}\right)-E_{i}^{2} \omega^{2}} \beta^{2}$

$-\sum_{i=1}^{n} \frac{\left(C_{i}\left(1+\omega^{2} B_{i}\right)-E_{i}\left(A_{i} \omega^{2}-\lambda\right)\right)}{D_{i}\left(1+\omega^{2} B_{i}\right)-E_{i}^{2} \omega^{2}} \beta+n$

$\frac{\partial}{\partial \omega^{2}} \mathcal{L}_{n}(\psi)=-\frac{1}{2} \sum_{i=1}^{n} \frac{D_{i} B_{i}-E_{i}^{2}}{D_{i}\left(1+\omega^{2} B_{i}\right)-E_{i}^{2} \omega^{2}}$

$+\sum_{i=1}^{n} \frac{2 A_{i}^{2}\left(1+\omega^{2} B_{i}\right)-2 B_{i}\left(\omega^{2} A_{i}^{2}+2 \lambda A_{i}-\lambda^{2} B_{i}\right)}{\left(2\left(1+\omega^{2} B_{i}\right)\right)^{2}}$

$-\frac{1}{2} \sum_{i=1}^{n} \frac{\left(\left(1+\omega^{2} B_{i}\right)\left(C_{i}-\beta\right)-E_{i}\left(A_{i} \omega^{2}+\lambda\right)\right)^{2}}{\left(1+\omega^{2} B_{i}\right)^{2}\left(D_{i}\left(1+\omega^{2} B_{i}\right)-E_{i}^{2} \omega^{2}\right)^{2}} \times$

$\left[\left(1+\omega^{2} B_{i}\right)\left(D_{i} B_{i}-E_{i}^{2}\right)+B_{i}\left(D_{i}\left(1+\omega^{2} B_{i}\right)-E_{i}^{2} \omega^{2}\right)\right]$

$+\sum_{i=1}^{n} \frac{\left[B_{i}\left(C_{i}-\beta\right)-A_{i} E_{i}\right]\left[\left(1+\omega^{2} B_{i}\right)\left(C_{i}-\beta\right)-E_{i}\left(A_{i} \omega^{2}+\lambda\right)\right]}{\left(1+\omega^{2} B_{i} D_{i}\right)\left(\left(1+\omega^{2} B_{i}\right)-E_{i}^{2} \omega^{2}\right)}$.

We study the estimators defined by the estimating function

$S_{n}\left(\hat{\psi}_{n}\right)=0$

Hence, the MLEs of $\psi_{0}=\left(\lambda_{0}, \omega_{0}^{2}, \beta_{0}\right)$ are given by the system:

$\hat{\lambda}_{n}=$

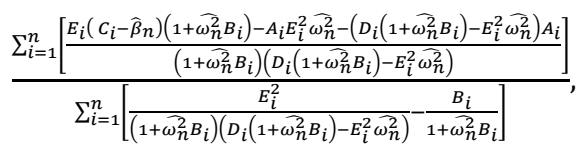

$\hat{\beta}_{n}=\frac{H_{i} \pm \sqrt{H_{i}^{2}-4 n K_{i}}}{2 K_{i}}$

Where,

$H_{i}=\sum_{i=1}^{n} \frac{C_{i}\left(1+\widehat{\omega_{n}^{2}} B_{i}\right)-E_{i}\left(A_{i} \widehat{\omega_{n}^{2}}-\widehat{\lambda}_{n}\right)}{D_{i}\left(1+\widehat{\omega}_{n}^{2} B_{i}\right)-E_{i}^{2} \widehat{\omega}_{n}^{2}}$

And

$K_{i}=\sum_{i=1}^{n} \frac{\left(1+\widehat{\omega_{n}^{2}} B_{i}\right)}{D_{i}\left(1+\widehat{\omega_{n}^{2}} B_{i}\right)-E_{i}^{2} \widehat{\omega_{n}^{2}}}$

$\sum_{i=1}^{n} \frac{D_{i} B_{i}-E_{i}^{2}}{D_{i}\left(1+\widehat{\left.\omega_{n}^{2} B_{i}\right)-E_{i}^{2} \widehat{\omega_{n}^{2}}}\right.}=$

$+\sum_{i=1}^{n} \frac{\left(\left(1+\widehat{\omega_{n}^{2}} B_{i}\right)\left(C_{i}-\widehat{\beta}_{n}\right)-E_{i}\left(A_{i} \widehat{\omega_{n}^{2}}+\widehat{\lambda}_{n}\right)\right)^{2}}{\left(1+\widehat{\omega_{n}^{2}} B_{i}\right)^{2}\left(D_{i}\left(1+\widehat{\omega_{n}^{2}} B_{i}\right)-E_{i}^{2} \widehat{\omega_{n}^{2}}\right)^{2}}$

$\times\left[\left(1+2 \widehat{\omega_{n}^{2}} B_{i}\right)\left(D_{i} B_{i}-E_{i}^{2}\right)+D_{i} E_{i}\right]$

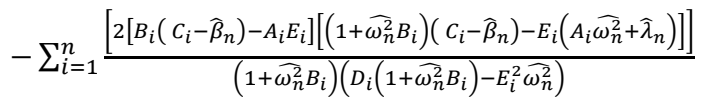

$-\sum_{i=1}^{n} \frac{A_{i}^{2}-2 \widehat{\lambda}_{n} A_{i} B_{i}+\widehat{\lambda}_{n}^{2} B_{i}^{2}}{2\left(1+\widehat{\omega}_{n}^{2} B_{i}\right)^{2}}$
For the fisher information matrix

$I(\psi)=$

$$
-\left(\begin{array}{ccc}
E_{\psi}\left(\frac{\partial^{2}}{\partial \lambda^{2}} \mathcal{L}_{n}(\psi)\right) & E_{\psi}\left(\frac{\partial^{2}}{\partial \lambda \partial \omega^{2}} \mathcal{L}_{n}(\psi)\right) & E_{\psi}\left(\frac{\partial^{2}}{\partial \lambda \partial \beta} \mathcal{L}_{n}(\psi)\right) \\
E_{\psi}\left(\frac{\partial^{2}}{\partial \omega^{2} \partial \lambda} \mathcal{L}_{n}(\psi)\right) & E_{\psi}\left(\frac{\partial^{2}}{\partial \omega^{2} \partial \omega^{2}} \mathcal{L}_{n}(\psi)\right) & E_{\psi}\left(\frac{\partial^{2}}{\partial \omega^{2} \partial \beta} \mathcal{L}_{n}(\psi)\right) \\
E_{\psi}\left(\frac{\partial^{2}}{\partial \beta \partial \lambda} \mathcal{L}_{n}(\psi)\right) & E_{\psi}\left(\frac{\partial^{2}}{\partial \beta \partial \omega^{2}} \mathcal{L}_{n}(\psi)\right) & E_{\psi}\left(\frac{\partial^{2}}{\partial \beta^{2}} \mathcal{L}_{n}(\psi)\right)
\end{array}\right),
$$

We get:

$\frac{\partial^{2}}{\partial \lambda^{2}} \mathcal{L}_{n}(\psi)=\sum_{i=1}^{n} \frac{E_{i}^{2}}{\left(1+\omega^{2} B_{i}\right)\left(D_{i}\left(1+\omega^{2} B_{i}\right)-E_{i}^{2} \omega^{2}\right)}-\frac{B_{i}}{1+\omega^{2} B_{i}}$

$\frac{\partial^{2}}{\partial \lambda \partial \beta} \mathcal{L}_{n}(\psi)=\sum_{i=1}^{n} \frac{E_{i}}{\left(D_{i}\left(1+\omega^{2} B_{i}\right)-E_{i}^{2} \omega^{2}\right)}$

$=\frac{\partial^{2}}{\partial \beta \partial \lambda} \mathcal{L}_{n}(\psi)$

$\frac{\partial^{2}}{\partial \lambda \partial \omega^{2}} \mathcal{L}_{n}(\psi)=\sum_{i=1}^{n} \frac{-\left(A_{i}-\lambda B_{i}\right) \mathrm{B}_{i}}{\left(1+\omega^{2} B_{i}\right)^{2}}$

$-\sum_{i=1}^{n} \frac{B_{i} E_{i}\left(C_{i}-\beta\right)-A_{i} E_{i}^{2}}{\left(1+\omega^{2} B_{i}\right)\left(D_{i}\left(1+\omega^{2} B_{i}\right)-E_{i}^{2} \omega^{2}\right)}$

$+\sum_{i=1}^{n} \frac{\left[\left(1+\omega^{2} B_{i}\right)\left(C_{i}-\beta\right) E_{i}-E_{i}^{2}\left(C_{i}-\beta\right)\right]}{\left(1+\omega^{2} B_{i}\right)^{2}\left(D_{i}\left(1+\omega^{2} B_{i}\right)-E_{i}^{2} \omega^{2}\right)^{2}}$

$\times\left[2 D_{i} B_{i}\left(1+\omega^{2} B_{i}\right)-E_{i}^{2}\left(1+2 \omega^{2} B_{i}\right)\right]$

After some elementary algebra we get:

$\frac{\partial^{2}}{\partial \lambda \partial \omega^{2}} \mathcal{L}_{n}(\psi)=\frac{\partial^{2}}{\partial \omega^{2} \partial \lambda} \mathcal{L}_{n}(\psi)$

$\frac{\partial^{2}}{\partial \beta^{2}} \mathcal{L}_{n}(\psi)=2 \beta K_{i}-H_{i}$

$\frac{\partial^{2}}{\partial \omega^{2} \partial \beta} \mathcal{L}_{n}(\psi)=$

$\sum_{i=1}^{n} \frac{-\left(1+\omega^{2} B_{i}\right)\left[B_{i}\left(C_{i}-\beta\right)-A_{i} E_{i}\right]}{\left(1+\omega^{2} B_{i}\right)\left(D_{i}\left(1+\omega^{2} B_{i}\right)-E_{i}^{2} \omega^{2}\right)}$

$-\sum_{i=1}^{n} \frac{\left[\left(1+\omega^{2} B_{i}\right)\left(C_{i}-\beta\right)-E_{i}\left(A_{i} \omega^{2}+\lambda\right)\right] B_{i}}{\left(1+\omega^{2} B_{i}\right)\left(D_{i}\left(1+\omega^{2} B_{i}\right)-E_{i}^{2} \omega^{2}\right)}$

$+\sum_{i=1}^{n} \frac{\left[\left(1+\omega^{2} B_{i}\right)\left(C_{i}-\beta\right)-E_{i}\left(A_{i} \omega^{2}+\lambda\right)\right]}{\left(1+\omega^{2} B_{i}\right)\left(D_{i}\left(1+\omega^{2} B_{i}\right)-E_{i}^{2} \omega^{2}\right)^{2}}$

$\times\left[\left(1+\omega^{2} B_{i}\right)\left(D_{i} B_{i}-E_{i}^{2}\right)+B_{i}\left(D_{i}\left(1+\omega^{2} B_{i}\right)-E_{i}^{2} \omega^{2}\right)\right]$

After some elementary algebra we get:

$\frac{\partial^{2}}{\partial \omega^{2} \partial \beta} \mathcal{L}_{n}(\psi)=\frac{\partial^{2}}{\partial \beta \partial \omega^{2}} \mathcal{L}_{n}(\psi)$

$\frac{\partial^{2}}{\partial \omega^{2} \partial \omega^{2}} \mathcal{L}_{n}(\psi)=\frac{1}{2} \sum_{i=1}^{n} \frac{\left(D_{i} B_{i}-E_{i}^{2}\right)^{2}}{\left(D_{i}\left(1+\omega^{2} B_{i}\right)-E_{i}^{2} \omega^{2}\right)^{2}}$

$-\sum_{i=1}^{n} \frac{B_{i}^{2}\left(\omega^{2} A_{i}^{2}+2 \lambda A_{i}-\lambda^{2} B_{i}\right)}{\left(1+\omega^{2} B_{i}\right)^{3}}$

$+\sum_{i=1}^{n} \frac{\left[B_{i}\left(C_{i}-\beta\right)-A_{i} E_{i}\right] B_{i}\left(C_{i}-\beta\right)}{\left(1+\omega^{2} B_{i}\right)\left(D_{i}\left(1+\omega^{2} B_{i}\right)-E_{i}^{2} \omega^{2}\right)}$

$-\sum_{i=1}^{n} \frac{\left[\left(1+\omega^{2} B_{i}\right)\left(C_{i}-\beta\right)-E_{i}\left(A_{i} \omega^{2}+\lambda\right)\right]^{2} B_{i}\left(D_{i} B_{i}-E_{i}^{2}\right)}{\left(1+\omega^{2} B_{i}\right)^{2}\left(D_{i}\left(1+\omega^{2} B_{i}\right)-E_{i}^{2} \omega^{2}\right)^{2}}$

$\times\left[2 D_{i} B_{i}\left(1+\omega^{2} B_{i}\right)-E_{i}^{2}\left(1-2 \omega^{2} B_{i}\right)\right]$ 
$\times\left[\frac{\left(D_{i} B_{i}-E_{i}^{2}\right)\left(1+\omega^{2} B_{i}\right)+\left(D_{i}\left(1+\omega^{2} B_{i}\right)-E_{i}^{2} \omega^{2}\right)\left(D_{i} B_{i}-E_{i}^{2}\right) B_{i}}{\left(1+\omega^{2} B_{i}\right)^{3}\left(D_{i}\left(1+\omega^{2} B_{i}\right)-E_{i}^{2} \omega^{2}\right)^{3}}\right]$.

(14)

\subsection{When $\varphi$ is random effect and $\mu$ is a parameter}

We introduce the distribution $Q_{\varphi, \mu}^{x^{i}, T_{i}}$ of $\left(X_{i}^{\varphi, \mu}(t), t \in\left[0, T_{i}\right]\right)$, where $\mu$ is unknown pararmeter.

Let $P_{\psi}^{i}=g(\varphi, \theta) d v(\varphi) \otimes Q_{\varphi}^{x^{i}, T_{i}}$ denote the joint distribution of $\left(\emptyset_{i}, X_{i}(t)\right)$ and let $Q_{\psi}^{i}$ denote the marginal distribution of $\left(X_{i}(t), t \in\left[0, T_{i}\right]\right)$. Let us consider the following assumption:

H3 For $i=1, \ldots, n$ and for all,$\varphi^{\prime}$,

$$
Q_{\varphi}^{x^{i}, T_{i}}\left(\int_{0}^{T_{i}} \frac{b^{2}\left(X_{i}^{\varphi, \mu}(t), \varphi^{\prime}\right)}{\sigma^{2}\left(X_{i}^{\varphi, \mu}(t)\right)} d t<+\infty\right)=1
$$

By the same approach of part one, we can derivative the joint density and estimators of the parameters (except there is no distribution of $\mu$ and the random effect $\varphi$ is normal) as follow:

$\gamma_{i}\left(X_{i}, \psi\right)=$

$\int_{\mathbb{R}} \exp \left(\varphi A_{i}-\frac{1}{2} \varphi^{2} B_{i}\right) \exp \left(\mu C_{i}-\right.$

$\left.\frac{1}{2} \mu^{2} D_{i}\right) \exp \left(-\varphi \mu E_{i}\right) g(\varphi, \theta) d v(\varphi)$

Hence,

$\gamma_{i}\left(X_{i}, \psi\right)=\frac{1}{\sqrt{1+\omega^{2} B_{i}}} \exp \left[\frac{-B_{i}}{2\left(1+\omega^{2} B_{i}\right)}\left(-\frac{\left(A_{i}-\mu E_{i}\right)}{B_{i}}\right)^{2}\right]$

$\times \exp \left(\frac{\left(A_{i}-\mu E_{i}\right)^{2}}{2 B_{i}}\right) \times \exp \left(\mu C_{i}-\frac{1}{2} \mu^{2} D_{i}\right)$.

And

$\mathcal{L}_{n}(\psi)=-\frac{1}{2} \sum_{i=1}^{n} \log \left(1+\omega^{2} B_{i}\right)$

$-\sum_{i=1}^{n} \frac{-B_{i}}{2\left(1+\omega^{2} B_{i}\right)}\left(\lambda-\frac{\left(A_{i}-\mu E_{i}\right)}{B_{i}}\right)^{2}$

$+\sum_{i=1}^{n} \frac{\left(A_{i}-\mu E_{i}\right)^{2}}{2 B_{i}}+\sum_{i=1}^{n}\left(\mu C_{i}-\frac{1}{2} \mu^{2} D_{i}\right)$

The estimators are given by the system:

$\hat{\lambda}_{n}=\sum_{i=1}^{n} \frac{A_{i}-\widehat{\mu}_{n} E_{i}}{1+\widehat{\omega}_{n}^{2} B_{i}} / \sum_{i=1}^{n} \frac{B_{i}}{1+\widehat{\omega}_{n}^{2} B_{i}}$

$\sum_{i=1}^{n}\left(\frac{A_{i}-\widehat{\mu}_{n} E_{i}}{1+\widehat{\omega}_{n}^{2} B_{i}}-\hat{\lambda}_{n} \frac{B_{i}}{1+\widehat{\omega}_{n}^{2} B_{i}}\right)^{2}=\sum_{i=1}^{n} \frac{B_{i}}{1+\widehat{\omega}_{n}^{2} B_{i}}$,

$\hat{\mu}_{n}=\frac{\sum_{i=1}^{n}\left[\frac{E_{i} \hat{\lambda}_{n} B_{i}-A_{i} E_{i}}{B_{i}\left(1+\widehat{\omega}_{n}^{2} B_{i}\right.}-\frac{A_{i} E_{i}}{B_{i}^{2}}+C_{i}\right]}{\sum_{i=1}^{n}\left[\frac{D_{i}^{2}}{B_{i}\left(1+\omega_{n}^{2} B_{i}\right)}-\frac{D_{i}^{2}}{B_{i}^{2}}+D_{i}\right]}$.

The fisher information matrix is,

$I(\psi)=$

$$
-\left(\begin{array}{ccc}
E_{\psi}\left(\frac{\partial^{2}}{\partial \lambda^{2}} \mathcal{L}_{n}(\psi)\right) & E_{\psi}\left(\frac{\partial^{2}}{\partial \lambda \partial \omega^{2}} \mathcal{L}_{n}(\psi)\right) & E_{\psi}\left(\frac{\partial^{2}}{\partial \lambda \partial \mu} \mathcal{L}_{n}(\psi)\right) \\
E_{\psi}\left(\frac{\partial^{2}}{\partial \omega^{2} \partial \lambda} \mathcal{L}_{n}(\psi)\right) & E_{\psi}\left(\frac{\partial^{2}}{\partial \omega^{2} \partial \omega^{2}} \mathcal{L}_{n}(\psi)\right) & E_{\psi}\left(\frac{\partial^{2}}{\partial \omega^{2} \partial \mu} \mathcal{L}_{n}(\psi)\right) \\
E_{\psi}\left(\frac{\partial^{2}}{\partial \mu \partial \lambda} \mathcal{L}_{n}(\psi)\right) & E_{\psi}\left(\frac{\partial^{2}}{\partial \mu \partial \omega^{2}} \mathcal{L}_{n}(\psi)\right) & E_{\psi}\left(\frac{\partial^{2}}{\partial \mu^{2}} \mathcal{L}_{n}(\psi)\right)
\end{array}\right),
$$

Where,

$$
\begin{aligned}
& \frac{\partial^{2}}{\partial \lambda^{2}} \mathcal{L}_{n}(\psi)=\sum_{i=1}^{n} \frac{B_{i}}{1+\omega^{2} B_{i}} \\
& \frac{\partial^{2}}{\partial \lambda \partial \omega^{2}} \mathcal{L}_{n}(\psi)=\sum_{i=1}^{n}\left[\frac{-B_{i}\left(A_{i}-\mu E_{i}\right)}{\left(1+\omega^{2} B_{i}\right)^{2}}+\lambda \frac{B_{i}^{2}}{\left(1+\omega^{2} B_{i}\right)^{2}}\right] \\
& =\frac{\partial^{2}}{\partial \omega^{2} \partial \lambda} \mathcal{L}_{n}(\psi) \\
& \frac{\partial^{2}}{\partial \lambda \partial \mu} \mathcal{L}_{n}(\psi)=\sum_{i=1}^{n} \frac{-E_{i}}{1+\omega^{2} B_{i}}=\frac{\partial^{2}}{\partial \mu \partial \lambda} \mathcal{L}_{n}(\psi) \\
& \frac{\partial^{2}}{\partial \omega^{2} \partial \omega^{2}} \mathcal{L}_{n}(\psi)=\sum_{i=1}^{n}\left[\left(\frac{-B_{i}\left(A_{i}-\mu E_{i}\right)}{\left(1+\omega^{2} B_{i}\right)^{2}}+\lambda \frac{B_{i}^{2}}{\left(1+\omega^{2} B_{i}\right)^{2}}\right)+\frac{B_{i}^{2}}{\left(1+\omega^{2} B_{i}\right)^{2}}\right] \\
& \frac{\partial^{2}}{\partial \omega^{2} \partial \mu} \mathcal{L}_{n}(\psi) \\
& =\sum_{i=1}^{n}\left[\left(\frac{A_{i}-\mu E_{i}}{1+\omega^{2} B_{i}}+\lambda \frac{B_{i}}{1+\omega^{2} B_{i}}\right) \frac{-E_{i}}{1+\omega^{2} B_{i}}\right] \\
& =\frac{\partial^{2}}{\partial \mu \partial \omega^{2}} \mathcal{L}_{n}(\psi) \\
& \frac{\partial^{2}}{\partial \mu^{2}} \mathcal{L}_{n}(\psi)=\sum_{i=1}^{n}\left[\frac{E_{i}^{2}}{B_{i}^{2}}-\frac{E_{i}^{2}}{\left(1+\omega^{2} B_{i}\right) B_{i}}-D_{i}\right] .
\end{aligned}
$$

\subsection{When $\varphi$ is random effect with normal distribution and $\mu$ is a constant $(\mu>0)$.}

From equation (15), we have the estimators for the parameters $\lambda$ and $\omega^{2}$ by the system:

$\hat{\lambda}_{n}=\sum_{i=1}^{n} \frac{A_{i}-\mu E_{i}}{1+\widehat{\omega}_{n}^{2} B_{i}} / \sum_{i=1}^{n} \frac{B_{i}}{1+\widehat{\omega_{n}^{2} B_{i}}}$,

$\sum_{i=1}^{n}\left(\frac{A_{i}-\mu E_{i}}{1+\widehat{\omega}_{n}^{2} B_{i}}-\hat{\lambda}_{n} \frac{B_{i}}{1+\widehat{\omega}_{n}^{2} B_{i}}\right)^{2}=\sum_{i=1}^{n} \frac{B_{i}}{1+\widehat{\omega}_{n}^{2} B_{i}}$

By suppose that $F_{i}=A_{i}-\mu E_{i}$,

$\hat{\lambda}_{n}=\sum_{i=1}^{n} \frac{F_{i}}{1+\widehat{\omega}_{n}^{2} B_{i}} / \sum_{i=1}^{n} \frac{B_{i}}{1+\widehat{\omega_{n}^{2} B_{i}},}$

$\sum_{i=1}^{n}\left(\frac{F_{i}}{1+\widehat{\omega}_{n}^{2} B_{i}}-\hat{\lambda}_{n} \frac{B_{i}}{1+\widehat{\omega}_{n}^{2} B_{i}}\right)^{2}=\sum_{i=1}^{n} \frac{B_{i}}{1+\widehat{\omega}_{n}^{2} B_{i}}$

In order to studying the maximum likelihood estimator of $\psi=\left(\lambda, \omega^{2}\right)$, we consider properties of the following random variables:

$\mathcal{J}_{i}(\psi)=\frac{F_{i}-\lambda B_{i}}{1+\omega^{2} B_{i}}, \mathcal{S}_{i}\left(\omega^{2}\right)=\frac{B_{i}}{1+\omega^{2} B_{i}}$

The score function is

$\frac{\partial}{\partial \lambda} \mathcal{L}_{n}(\psi)=\sum_{i=1}^{n} \mathcal{J}_{i}(\psi)$,

$\frac{\partial}{\partial \omega^{2}} \mathcal{L}_{n}(\psi)=\frac{1}{2} \sum_{i=1}^{n}\left(\mathcal{J}_{i}^{2}(\psi)-\mathcal{S}_{i}\left(\omega^{2}\right)\right)$

We need the following lemma to study an important moments of $\mathcal{J}_{i}(\psi), \mathcal{S}_{i}\left(\omega^{2}\right)$.

Lemma 1: For all $\psi=\left(\lambda, \omega^{2}\right) \in \mathbb{R} \times \mathbb{R}^{+}$and all $h \in \mathbb{R}$,

$E_{\psi}\left(\exp \left(h \frac{F_{1}}{1+\omega^{2} B_{1}}\right)\right)<+\infty$.

Proof: From (17), we set $\mathcal{J}_{1}(\psi)=\mathcal{J}_{1}$ and $\mathcal{S}_{1}\left(\omega^{2}\right)=\mathcal{S}_{1}$. Let $l\left(X_{1}, \psi\right)=\log \gamma_{1}\left(X_{1}, \psi\right)$ and set $\psi(h)=\left(\lambda+h, \omega^{2}\right)$, then:

$l\left(X_{1}, \psi(h)\right)=\log \gamma_{1}\left(X_{1}, \psi\right)$ 
$=-\frac{1}{2} \log \left(1+\omega^{2} B_{i}\right)$

$-\frac{B_{i}}{2\left(1+\omega^{2} B_{i}\right)}\left(\lambda-\frac{\left(A_{i}-\mu E_{i}\right)}{B_{i}}\right)^{2}+\frac{\left(A_{i}-\mu E_{i}\right)^{2}}{2 B_{i}}$

$+\left(\mu C_{i}-\frac{1}{2} \mu^{2} D_{i}\right)$

$=l\left(X_{1}, \psi\right)+h \frac{F_{i}-\lambda B_{1}}{1+\omega^{2} B_{1}}-\frac{F_{1}^{2}}{2} \mathcal{S}_{1}+\left(\mu C_{1}-\frac{1}{2} \mu^{2} D_{1}\right)$

$=l\left(X_{1}, \psi\right)+h \mathcal{J}_{1}-\frac{h^{2}}{2} \delta_{1}+\left(\mu C_{1}-\frac{1}{2} \mu^{2} D_{1}\right)$.

The first and second derivative w.r.t $\lambda$ are given as follow,

$\frac{\partial}{\partial \lambda} l\left(X_{1}, \psi\right)=-\frac{B_{i}}{\left(1+\omega^{2} B_{1}\right)}\left(\lambda-\frac{F_{i}}{B_{1}}\right)=\mathcal{J}_{1}$

, and

$\frac{\partial^{2}}{\partial \lambda^{2}} l\left(X_{1}, \psi\right)=-\mathcal{S}_{1}$.

Hence,

$\gamma_{1}\left(X_{1}, \psi\right) \exp \left(h \mathcal{J}_{1}\right)=l\left(X_{1}, \psi(h)\right) \exp \left(\frac{h^{2}}{2} \delta_{1}\right)$

And since $\mathcal{S}_{1} \leq \frac{1}{\omega^{2}}$

$E_{\psi} \exp \left(h \mathcal{J}_{1}\right)=E_{\psi(h)} \exp \left(\frac{h^{2}}{2} \delta_{1}\right)$

$\leq \exp \left(\frac{h^{2}}{2 \omega^{2}}\right)$

$<+\infty$,

Then,

$E_{\psi}\left(\exp \left(h \frac{F_{1}}{1+\omega^{2} B_{1}}\right)\right) \leq E_{\psi} \exp \left(h \mathcal{J}_{1}\right) \exp \left(\frac{(h+\lambda)^{2}}{4 \omega^{2}}\right)$

$$
<+\infty
$$

Proposition 3.2 For all $\psi=\left(\lambda, \omega^{2}\right) \in \mathbb{R} \times \mathbb{R}^{+}$, the following relations hold:

$$
\begin{aligned}
& E_{\psi}\left(\mathcal{J}_{1}(\psi)\right)=0, E_{\psi}\left(\mathcal{J}_{1}^{2}(\psi)\right)=E_{\psi}\left(\mathcal{S}_{1}\left(\omega^{2}\right)\right) \\
& E_{\psi}\left(\mathcal{J}_{1}^{3}(\psi)\right)=3 E_{\psi}\left(\mathcal{J}_{1}(\psi) \mathcal{S}_{1}\left(\omega^{2}\right)\right) \\
& E_{\psi}\left(\mathcal{J}_{1}^{2}(\psi)-\mathcal{S}_{1}\left(\omega^{2}\right)\right)^{2} \\
& =4 E_{\psi}\left(\mathcal{J}_{1}^{2}(\psi) \mathcal{S}_{1}\left(\omega^{2}\right)\right)-2 E_{\psi}\left(\mathcal{S}_{1}^{2}\left(\omega^{2}\right)\right) .
\end{aligned}
$$

Proof: We set $\mathcal{J}_{1}(\psi)=\mathcal{J}_{1}$ and $\mathcal{S}_{1}\left(\omega^{2}\right)=\mathcal{S}_{1}$. Let $\psi=\left(\lambda, \omega^{2}\right)$ and $\tau=\left(0, \omega^{2}\right)$ and from the relation:

$\gamma_{i}\left(X_{i}, \psi\right)=\frac{d Q_{\psi}^{i}}{d Q^{i}}\left(X_{i}\right)$

We set

$$
\begin{aligned}
& g_{1}(\psi)=\frac{\gamma_{1}\left(X_{1}, \psi\right)}{\gamma_{1}\left(X_{1}, \tau\right)}=\frac{d Q_{\psi}^{1}}{d Q^{1}} / \frac{d Q_{\tau}^{1}}{d Q^{1}}=\frac{d Q_{\psi}^{1}}{d Q_{\tau}^{1}} \\
& =\exp \left(\lambda \frac{F_{1}}{1+\omega^{2} B_{1}}-\frac{\lambda^{2} B_{1}}{2\left(1+\omega^{2} B_{1}\right)}\right),
\end{aligned}
$$

So that

$\int_{C_{T}} g_{1}(\psi) d Q_{\tau}^{1}=1$

Provided that we can swap derivation with respect to $\lambda$ and integration with respect to $Q_{\tau}^{1}$,

$\int_{C_{T}} \frac{\partial^{j} g_{1}}{\partial \lambda^{j}}(\psi) d Q_{\tau}^{1}=0$

Hold for $j \geq 1$, where $C_{T}$ denote the space of all real continuous functions $(x(t), t \in[0, T])$ defined on $[0, T]$.

For $=1,2,3,4$, the moments relations hold from (19) as follow:

$\frac{\partial g_{1}}{\partial \lambda}(\psi)=\mathcal{J}_{1} g_{1}(\psi), \frac{\partial^{2} g_{1}}{\partial \lambda^{2}}(\psi)=\left(\mathcal{J}_{1}^{2}-\mathcal{S}_{1}\right) g_{1}(\psi)$,

$\frac{\partial^{3} g_{1}}{\partial \lambda^{3}}(\psi)=\left(\mathcal{J}_{1}^{3}-3 \mathcal{J}_{1} \mathcal{S}_{1}\right) g_{1}(\psi)$,

$\frac{\partial^{4} g_{1}}{\partial \lambda^{4}}(\psi)=\left(\mathcal{J}_{1}^{4}-6 \mathcal{J}_{1}^{2} \mathcal{S}_{1}+3 \mathcal{S}_{1}^{2}\right) g_{1}(\psi)$

Then,

$\int_{C_{T}} \frac{\partial g_{1}}{\partial \lambda}(\psi) d Q_{\tau}^{1}=\int_{C_{T}} \mathcal{J}_{1} g_{1}(\psi) d Q_{\tau}^{1}=0$ yield, $E_{\psi}\left(\mathcal{J}_{1}(\psi)\right)=0$,

$\frac{\partial^{2} g_{1}}{\partial \lambda^{2}}(\psi) d Q_{\tau}^{1}=\int_{C_{T}}\left(\mathcal{J}_{1}^{2}-\mathcal{S}_{1}\right) g_{1}(\psi) d Q_{\tau}^{1}$ yields, $E_{\psi}\left(\mathcal{J}_{1}^{2}(\psi)\right)=$ $E_{\psi}\left(\delta_{1}\right)$,

$\frac{\partial^{3} g_{1}}{\partial \lambda^{3}}(\psi) d Q_{\tau}^{1}=\int_{C_{T}}\left(\mathcal{J}_{1}^{3}-3 \mathcal{J}_{1} \mathcal{S}_{1}\right) g_{1}(\psi) d Q_{\tau}^{1}$ yield, $E_{\psi}\left(\mathcal{J}_{1}^{3}(\psi)\right)=$ $3 E_{\psi}\left(\mathcal{J}_{1}(\psi) \mathcal{S}_{1}\left(\omega^{2}\right)\right)$,

And, $\frac{\partial^{4} g_{1}}{\partial \lambda^{4}}(\psi) d Q_{\tau}^{1}=\int_{C_{T}}\left(\mathcal{J}_{1}^{4}-6 \mathcal{J}_{1}^{2} \mathcal{S}_{1}+3 \mathcal{S}_{1}^{2}\right) g_{1}(\psi) d Q_{\tau}^{1}$ yield,

$E_{\psi}\left(\mathcal{J}_{1}^{2}(\psi)-\mathcal{S}_{1}\left(\omega^{2}\right)\right)^{2}=4 E_{\psi}\left(\mathcal{J}_{1}^{2}(\psi) \mathcal{S}_{1}\left(\omega^{2}\right)\right)$

$-2 E_{\psi}\left(\mathcal{S}_{1}^{2}\left(\omega^{2}\right)\right)$

For justify the swap of derivation and integration. Let us fix $\bar{\lambda}$ and $\epsilon>0$. For $\lambda \in[\bar{\lambda}-\epsilon, \bar{\lambda}+\epsilon]$, we have the bound

$\left|\frac{\partial g_{1}}{\partial \lambda}(\psi)\right| \leq\left(\left|\frac{F_{1}}{1+\omega^{2} B_{1}}\right|+\frac{C}{\omega^{2}}\right)\left(\exp \left((\bar{\lambda}-\epsilon) \frac{F_{1}}{1+\omega^{2} B_{1}}\right)+\right.$ $\left.\exp \left((\bar{\lambda}+\epsilon) \frac{F_{1}}{1+\omega^{2} B_{1}}\right)\right)$

Where $C=|\bar{\lambda}-\epsilon|+|\bar{\lambda}+\epsilon|$.

The upper bound is independent of $\lambda$ and integrable w.r.t. $Q_{\tau}^{1}$ by lemma 1 , by the same way we investigate the other derivatives.

Remark 1: From (17) we get, $\frac{\partial \mathcal{J}_{i}}{\partial \lambda}=-\mathcal{S}_{i}, \frac{\partial \mathcal{J}_{i}}{\partial \omega^{2}}=-\mathcal{J}_{i} \mathcal{S}_{i}$ and $\frac{\partial \mathcal{S}_{i}}{\partial \omega^{2}}=$ $-\delta_{i}^{2}\left(\omega^{2}\right)$.

Depending on the law of large number, CLT, lemma 1, remark1 and the result of proposition3.2,

The random vector

$v=\frac{1}{\sqrt{n}}\left(\frac{\partial}{\partial \lambda} \mathcal{L}_{n}(\psi) \frac{\partial}{\partial \omega^{2}} \mathcal{L}_{n}(\psi)\right)^{\prime}=$

$\frac{1}{\sqrt{n}}\left(\sum_{i=1}^{n} \mathcal{J}_{i}(\psi) \frac{1}{2} \sum_{i=1}^{n}\left(\mathcal{J}_{i}^{2}(\psi)-\mathcal{S}_{i}\left(\omega^{2}\right)\right)\right)^{\prime}$

Converge in distribution to $\mathcal{N}_{2}(0, I(\psi))$ for all, under $Q_{\psi}$, as $n$ goes to infinity. 
The matrix

$-\frac{1}{n}\left(\begin{array}{cc}\frac{\partial^{2}}{\partial \lambda^{2}} \mathcal{L}_{n}(\psi) & \frac{\partial^{2}}{\partial \lambda \partial \omega^{2}} \mathcal{L}_{n}(\psi) \\ \frac{\partial^{2}}{\partial \omega^{2} \partial \lambda} \mathcal{L}_{n}(\psi) & \frac{\partial^{2}}{\partial \omega^{2} \partial \omega^{2}} \mathcal{L}_{n}(\psi)\end{array}\right)$

Converge in probability to $I(\psi)$, where

$\frac{\partial^{2}}{\partial \lambda^{2}} \mathcal{L}_{n}(\psi)=-\sum_{i=1}^{n} \delta_{i}\left(\omega^{2}\right)$

$\frac{\partial^{2}}{\partial \lambda \partial \omega^{2}} \mathcal{L}_{n}(\psi)=-\sum_{i=1}^{n} \mathcal{J}_{i}(\psi) \mathcal{S}_{i}\left(\omega^{2}\right)$

$\frac{\partial^{2}}{\partial \omega^{2} \partial \omega^{2}} \mathcal{L}_{n}(\psi)$

$-\frac{1}{2} \sum_{i=1}^{n}\left(2 \mathcal{J}_{1}^{2}(\psi) \mathcal{S}_{1}\left(\omega^{2}\right)-\mathcal{S}_{1}^{2}\left(\omega^{2}\right)\right)$

And

$I(\psi)=$

$\left(\begin{array}{cc}E_{\psi}\left(\mathcal{S}_{1}\left(\omega^{2}\right)\right) & E_{\psi}\left(\mathcal{J}_{1}(\psi) \mathcal{S}_{1}\left(\omega^{2}\right)\right) \\ E_{\psi}\left(\mathcal{J}_{1}(\psi) \mathcal{S}_{1}\left(\omega^{2}\right)\right) & E_{\psi}\left(\mathcal{J}_{1}^{2}(\psi) \mathcal{S}_{1}\left(\omega^{2}\right)\right)-\frac{1}{2} E_{\psi}\left(\mathcal{S}_{1}^{2}\left(\omega^{2}\right)\right)\end{array}\right)$,

Is the covariance matrix of the vector

$\left(\mathcal{J}_{i}(\psi) \frac{1}{2}\left(\mathcal{J}_{i}^{2}(\psi)-\mathcal{S}_{i}\left(\omega^{2}\right)\right)\right)^{\prime}$

\section{Asymptotic properties of the estimators}

In this section we focus on the consistency and asymptotic normality when the random effect $\varphi$ is normal and $\mu$ is constant.

We need the following assumptions in order to prove the properties of estimators:

H4 the parameter set $\Theta$ is a compact subset of $\mathbb{R} \times \mathbb{R}^{+}$.

H5 the true value $\psi_{0}$ belongs to $\operatorname{int}(\Theta)$.

H6 $I\left(\psi_{0}\right)$ is invertible.

\subsection{Strong consistency}

Proposition4.1 Under $\mathrm{H} 1-\mathrm{H} 3, Q_{\psi_{0}}^{1}=Q_{\psi}^{1}$ implies that, $\theta_{0}=\theta$ Hence, $\psi \rightarrow K\left(Q_{\psi_{0}}^{1}, Q_{\psi}^{1}\right)$ admits a unique minimum at $\psi_{0}=\psi$, where $K\left(Q_{\psi_{0}}^{1}, Q_{\psi}^{1}\right)$ is the kullback information of $Q_{\psi_{0}}^{1}$ w.r.t $Q_{\psi}^{1}$.

Proof: By analogue way of proof of proposition7 in [24].

Proposition4.2 Under $\mathrm{H} 1-\mathrm{H} 3$ and $\mathrm{H} 4$ and under $Q_{\psi_{0}}, \widehat{\psi}_{n}$ converges in probability to $\psi_{0}$, where $\hat{\psi}_{n}$ is the maximum likelihood estimator defined as any solution of $\mathcal{L}_{n}\left(\hat{\psi}_{n}\right)=\sup _{\psi \in \Theta} \mathcal{L}_{n}(\psi)$.

Proof: Since $\frac{1}{n}\left(\mathcal{L}_{n}\left(\psi_{0}\right)-\mathcal{L}_{n}(\psi)\right)$ converges in probability to $K\left(Q_{\psi_{0}}^{1}, Q_{\psi}^{1}\right)$, the likelihood $-\frac{1}{n} \mathcal{L}_{n}(\psi)$ is a contrast process with $\psi \rightarrow K\left(Q_{\psi_{0}}^{1}, Q_{\psi}^{1}\right)$, by the standard proof of consistency (van der vaart 2000 [26]).

Now we will proof the continuity of $-\frac{1}{n} \mathcal{L}_{n}(\psi)$ :

Define

$w_{n}(\delta)=\sup _{\left\|\psi-\psi^{\prime}\right\| \leq \delta, \psi, \psi^{\prime} \in \Theta}\left|\mathcal{L}_{n}(\psi)-\mathcal{L}_{n}\left(\psi^{\prime}\right)\right| / n$

Let $w_{n}(\delta) \leq \sup _{\psi \in \Theta}\left\|\nabla \mathcal{L}_{n}(\psi) / n\right\|$ and bounded the score function (18).by H4, we have

$\Theta \subset[\underline{\lambda}, \bar{\lambda}] \times\left[\underline{\omega^{2}}, \overline{\omega^{2}}\right]$ With $\underline{\lambda}<\bar{\lambda}, 0<\underline{\omega^{2}}<\overline{\omega^{2}}$.
We have

$\zeta_{i}(\psi)=\frac{F_{i}}{1+\omega_{0}^{2} B_{i}}\left(1+\frac{\left(\omega_{0}^{2}-\omega^{2}\right) B_{i}}{1+\omega^{2} B_{i}}\right)-\lambda \frac{B_{i}}{1+\omega^{2} B_{i}}$.

Then,

$\sup _{\psi \in \Theta}\left|\zeta_{i}(\psi)\right| \leq\left|\frac{F_{i}}{1+\omega_{0}^{2} B_{i}}\right|\left(2+\frac{\omega_{0}^{2}}{\underline{\omega^{2}}}\right)+\frac{|\bar{\lambda}|}{\underline{\omega^{2}}}$.

There is a constant $K$ such that

$E_{\psi_{0}} w_{n}(\delta) \leq K \delta E_{\psi_{0}}\left(\left|\frac{F_{1}}{1+\omega_{0}^{2} B_{1}}\right|+\left(\frac{F_{1}}{1+\omega_{0}^{2} B_{1}}\right)^{2}\right)$.

Hence, the consistency of $\widehat{\psi}_{n}$ hold.

\subsection{Asymptotic normality}

Proposition4.3 Assume H1-H3, the function

$\psi \rightarrow K\left(Q_{\psi_{0}}^{1}, Q_{\psi}^{1}\right)$ is continuous on $\mathbb{R} \times \mathbb{R}^{+}$.

Proof: From (15), let $\mathcal{L}_{1}(\psi)=\gamma_{1}\left(X_{1}, \psi\right)$, then

$E_{\psi_{0}}\left(\log \frac{\gamma_{1}\left(X_{1}, \psi_{0}\right)}{\gamma_{1}\left(X_{1}, \psi\right)}\right)=K\left(Q_{\psi_{0}}^{1}, Q_{\psi}^{1}\right)$

$=E_{\psi_{0}}\left(\mathcal{L}_{1}\left(\psi_{0}\right)-\mathcal{L}_{1}(\psi)\right)$

We obtain

$\mathcal{L}_{1}\left(\psi_{0}\right)-\mathcal{L}_{1}(\psi)=\frac{1}{2} \log \left(\frac{1+\omega^{2} B_{1}}{1+\omega_{0}^{2} B_{1}}\right)$

$+\frac{1}{2} \frac{\left(\omega_{0}^{2}-\omega^{2}\right) F_{1}^{2}}{\left(1+\omega^{2} B_{1}\right)\left(1+\omega_{0}^{2} B_{1}\right)}+\frac{\lambda^{2} B_{1}}{2\left(1+\omega^{2} B_{1}\right)}$

$-\frac{\lambda F_{1}}{1+\omega^{2} B_{1}}-\left(\frac{\lambda_{0}^{2} B_{1}}{2\left(1+\omega_{0}^{2} B_{1}\right)}-\frac{\lambda_{0} F_{1}}{1+\omega_{0}^{2} B_{1}}\right)$

Now, we show that this random variable has finite expectation. Let us consider the upper bound:

$0<\frac{1+\omega^{2} B_{1}}{1+\omega_{0}^{2} B_{1}}<1+\frac{\omega^{2}}{\omega_{0}^{2}}$

From the relation $(x)=x-1-\log x$, which is non-negative and define on $\mathbb{R}^{+}$, we get the lower bound:

$f\left(\frac{1+\omega_{0}^{2} B_{1}}{1+\omega^{2} B_{1}}\right)=\frac{1+\omega_{0}^{2} B_{1}}{1+\omega^{2} B_{1}}-1-\log \left(\frac{1+\omega_{0}^{2} B_{1}}{1+\omega^{2} B_{1}}\right)$

$\log \left(F_{1}\right)=f\left(\frac{1+\omega_{0}^{2} B_{1}}{1+\omega^{2} B_{1}}\right)+\left(\omega^{2}-\omega_{0}^{2}\right) \frac{B_{1}}{1+\omega^{2} B_{1}}$

$\geq\left(\omega^{2}-\omega_{0}^{2}\right) \frac{B_{1}}{1+\omega^{2} B_{1}}$

So that

$\left|\log \left(\frac{1+\omega^{2} B_{1}}{1+\omega_{0}^{2} B_{1}}\right)\right| \leq \log \left(1+\frac{\omega^{2}}{\omega_{0}^{2}}\right)+\frac{\left|\omega^{2}-\omega_{0}^{2}\right|}{\omega^{2}}$

And

$$
\begin{aligned}
0 & <\frac{F_{1}^{2}}{\left(1+\omega^{2} B_{1}\right)\left(1+\omega_{0}^{2} B_{1}\right)}=\left(\frac{F_{1}}{1+\omega_{0}^{2} B_{1}}\right)^{2} \frac{1+\omega_{0}^{2} B_{1}}{1+\omega^{2} B_{1}} \\
& \leq\left(\frac{F_{1}}{1+\omega_{0}^{2} B_{1}}\right)^{2}\left(1+\frac{\omega^{2}}{\omega_{0}^{2}}\right)
\end{aligned}
$$

Has finite expectation due to lemma1. 
Also,

$\frac{F_{1}}{1+\omega^{2} B_{1}}=\frac{F_{1}}{1+\omega_{0}^{2} B_{1}}\left(1+\left(\omega_{0}^{2}-\omega^{2}\right) \frac{B_{1}}{1+\omega^{2} B_{1}}\right)$

Then

$\left|\frac{F_{1}}{1+\omega^{2} B_{1}}\right| \leq\left|\frac{F_{1}}{1+\omega_{0}^{2} B_{1}}\right|\left(1+\frac{\left|\omega_{0}^{2}-\omega^{2}\right|}{\omega^{2}}\right)$.

Has finite expectation under $E_{\psi_{0}}$ by lemma1

From above and for all $=\left(\lambda, \omega^{2}\right) \in[\underline{\lambda}, \bar{\lambda}] \times\left[\underline{\omega^{2}}, \overline{\omega^{2}}\right] \subset \mathbb{R} \times \mathbb{R}^{+}$, the term $\left|\mathcal{L}_{1}\left(\psi_{0}\right)-\mathcal{L}_{1}(\psi)\right|$ has finite $E_{\psi_{0}}$-expectation which means $K\left(Q_{\psi_{0}}^{1}, Q_{\psi}^{1}\right)$ is continuous. Hence the result.

Proposition4.4: Under H1-H3 and $\mathrm{H} 4-\mathrm{H} 6$, as $n$ tends to infinity, the maximum likelihood estimator satisfies $\sqrt{n}\left(\hat{\psi}_{n}-\right.$ $\left.\psi_{0}\right) \rightarrow^{D} \mathcal{N}_{2}\left(0, I^{-1}\left(\psi_{0}\right)\right)$.

Proof: Let $\hat{\psi}_{n, i}, \psi_{0, i}$ be the components of $\hat{\psi}_{n}, \psi_{0}$, assume that

$A_{n}(\psi)=-\frac{1}{n} \mathcal{L}_{n}(\psi)$

And

$A_{n, i}^{\prime}=-\frac{1}{n} \frac{\partial}{\partial \psi_{i}} \mathcal{L}_{n}(\psi)$ And $A_{n, i j}^{\prime \prime}=-\frac{1}{n} \frac{\partial^{2}}{\partial \psi_{i} \partial \psi_{i}} \mathcal{L}_{n}(\psi)$

From the proof of consistency and assumption (5), $Q_{\psi_{0}}\left(\hat{\psi}_{n} \in\right.$ $\operatorname{int}(\Theta)) \rightarrow 1$.

By using Taylor formula, we get:

$0=A_{n, i}^{\prime}\left(\hat{\psi}_{n}\right)$

$=A_{n, i}^{\prime}\left(\psi_{0}\right)+\sum_{j=1,2}\left(\hat{\psi}_{n, j}-\psi_{0, j}\right)\left(A_{n, i j}^{\prime \prime}\left(\psi_{0}\right)+R_{n}\right)$,

Where

$R_{n}=\int_{0}^{1}\left(A_{n, i j}^{\prime \prime}\left(\psi_{0}+s\left(\hat{\psi}_{n}-\psi_{0}\right)\right)-A_{n, i j}^{\prime \prime}\left(\psi_{0}\right)\right) d s$

We must prove that $R_{n}$ goes to zero in probability. Using (20)(21), compute the derivatives as follow:

$\frac{1}{n} \frac{\partial^{3}}{\partial \lambda^{3}} \mathcal{L}_{n}(\psi)=0$,

$\frac{1}{n} \frac{\partial^{3}}{\partial \omega^{2} \partial \omega^{2} \partial \omega^{2}} \mathcal{L}_{n}(\psi)=\frac{1}{n} \sum_{i=1}^{n}\left[3 \mathcal{J}_{i}^{2}(\psi) \mathcal{S}_{1}^{2}\left(\omega^{2}\right)-\mathcal{S}_{1}^{3}\left(\omega^{2}\right)\right]$,

$\frac{1}{n} \frac{\partial^{3}}{\partial^{2} \lambda \partial \omega^{2}} \mathcal{L}_{n}(\psi)=\frac{1}{n} \sum_{i=1}^{n} \delta_{i}^{2}\left(\omega^{2}\right)$,

$\frac{1}{n} \frac{\partial^{3}}{\partial \lambda \partial \omega^{2} \partial \omega^{2}} \mathcal{L}_{n}(\psi)=\frac{2}{n} \sum_{i=1}^{n} \mathcal{J}_{i}(\psi) \mathcal{S}_{1}^{2}\left(\omega^{2}\right)$.

From (22), for a constant $K$, we obtain

$\left|R_{n}\right| \leq K\left|\hat{\psi}_{n}-\psi_{0}\right| \frac{1}{n} \sum_{i=1}^{n}\left(1+\left(\left|\frac{F_{1}}{1+\omega_{0}^{2} B_{1}}\right|+\left(\frac{F_{1}}{1+\omega_{0}^{2} B_{1}}\right)^{2}\right)\right)$,

By using the proof of Proposition 4.2. , $R_{n}$ tends to zero. This means the result.

\section{Conclusion}

We depend on SDE with random effects model framework and consider the linearity assumption in the drift function given by: i) $\quad b\left(x, \emptyset_{i}, \mu_{i}\right)=\emptyset_{i} b\left(X_{i}(t)\right)+\mu_{i}$ where $\emptyset_{i}$ are supposed to be Gaussian random variables with mean $\lambda$ and variance $\omega^{2}$, and $\mu_{i}$ to be exponential random variables with parameter $\beta$

ii) $\quad b\left(x, \emptyset_{i}, \mu_{i}\right)=\emptyset_{i} b\left(X_{i}(t)\right)+\mu \quad$ where $\emptyset_{\mathrm{i}} \quad$ are $\operatorname{an}\left(\lambda, \omega^{2}\right)$ and $\mu$ is unknown parameter,

iii) $\quad b\left(x, \emptyset_{i}, \mu_{i}\right)=\emptyset_{i} b\left(X_{i}(t)\right)+\mu$ Where $\emptyset_{\mathrm{i}}$ are Gaussian $\left(\lambda, \omega^{2}\right)$ and $\mu$ is a constant. A closed form expression of the likelihoods of the parameters of the i.i.d random effects and the maximum likelihood estimator are obtained. We proved consistency and asymptotic normality of the estimators in the third case only.

\section{References}

[1] E. Allen, Modeling with Itô stochastic differential equations, Springer Science \& Business Media, 2007.

[2] R. Hindriks, Empirical dynamics of neuronal rhythms, in, Ph. D. Thesis, VU University Amsterdam, 2011.

[3] M. Musiela, M. Rutkowski, Martingale methods in financial modelling, Springer Science \& Business Media, 2006.

[4] S. Gugushvili, P. Spreij, Parametric inference for stochastic differential equations: a smooth and match approach, arXiv preprint arXiv: 1111. 1120, (2011).

[5] E. Wong, B. Hajek, Stochastic processes in engineering systems, Springer Science \& Business Media, 2012.

[6] J.N. Nielsen, H. Madsen, P.C. Young, Parameter estimation in stochastic differential equations: an overview, Annual Reviews in Control, 24 (2000) 83-94. http://dx.doi.org/10.1016/S13675788(00)90017-8.

[7] G.B. Durham, A.R. Gallant, Numerical techniques for maximum likelihood estimation of continuous-time diffusion processes, Journal of Business \& Economic Statistics, 20 (2002) 297-338. http://dx.doi.org/10.1198/073500102288618397.

[8] P.D. Feigin, Maximum likelihood estimation for continuous-time stochastic processes, Advances in Applied Probability, (1976) 712736. http://dx.doi.org/10.1017/S0001867800042890.

[9] R.V. Overgaard, N. Jonsson, C.W. Tornøe, H. Madsen, Non-linear mixed-effects models with stochastic differential equations: implementation of an estimation algorithm, Journal of pharmacokinetics and pharmacodynamics, 32 (2005) 85-107. http://dx.doi.org/10.1007/s10928-005-2104-x.

[10] C.W. Tornøe, R.V. Overgaard, H. Agers $\varnothing$, H.A. Nielsen, H. Madsen, E.N. Jonsson, Stochastic differential equations in NONMEM®: implementation, application, and comparison with ordinary differential equations, Pharmaceutical research, 22 (2005) 1247-1258. http://dx.doi.org/10.1007/s11095-005-5269-5.

[11] M. Delattre, M. Lavielle, Coupling the SAEM algorithm and the extended Kalman filter for maximum likelihood estimation in mixed-effects diffusion models, Statistics and its interface, 6 (2013) 519--532. http://dx.doi.org/10.4310/SII.2013.v6.n4.a10.

[12] S. Klim, S.B. Mortensen, N.R. Kristensen, R.V. Overgaard, H. Madsen, Population stochastic modelling (PSM) — an R package for mixed-effects models based on stochastic differential equations, Computer methods and programs in biomedicine, 94 (2009) 279 289. http://dx.doi.org/10.1016/j.cmpb.2009.02.001.0.

[13] S. Donnet, A. Samson, A review on estimation of stochastic differential equations for pharmacokinetic/pharmacodynamic models, Advanced Drug Delivery Reviews, 65 (2013) 929-939. http://dx.doi.org/10.1016/j.addr.2013.03.005.

[14] U. Picchini, A.D. GAETANO, S. Ditlevsen, Stochastic Differential Mixed-Effects Models, Scandinavian Journal of statistics, 37 (2010) 67-90. http://dx.doi.org/10.1111/j.1467-9469.2009.00665.x.

[15] W. Kampowsky, P. Rentrop, W. Schmidt, Classification and numerical simulation of electric circuits, (1992).

[16] U. Picchini, S. Ditlevsen, Practical estimation of high dimensional stochastic differential mixed-effects models, Computational Statistics \& Data Analysis, $55 \quad$ (2011) 1426-1444. http://dx.doi.org/10.1016/j.csda.2010.10.003.

[17] S. Beal, L. Sheiner, Estimating population kinetics, Critical reviews in biomedical engineering, 8 (1981) 195-222.

[18] A.R. Pedersen, A new approach to maximum likelihood estimation for stochastic differential equations based on discrete observations, Scandinavian journal of statistics, (1995) 55-71.

[19] M.W. Brandt, P. Santa-Clara, Simulated likelihood estimation of diffusions with an application to exchange rate dynamics in incomplete markets, Journal of financial economics, 63 (2002) 161-210. http://dx.doi.org/10.1016/S0304-405X(01)00093-9. 
[20] J. Nicolau, A new technique for simulating the likelihood of stochastic differential equations, The Econometrics Journal, 5 (2002) 91-103. http://dx.doi.org/10.1111/1368-423X.t01-1-00075.

[21] A. Hurn, K. Lindsay, Estimating the parameters of stochastic differential equations, Mathematics and computers in simulation, 48 (1999) 373-384. http://dx.doi.org/10.1016/S0378-4754(99)00017-8.

[22] A.W. Lo, Maximum likelihood estimation of generalized Itô processes with discretely sampled data, Econometric Theory, 4 (1988) 231-247. http://dx.doi.org/10.1017/S0266466600012044.

[23] Y. Ait-Sahalia, Maximum Likelihood Estimation of Discretely Sampled Diffusions: A Closed-form Approximation Approach, Econometrica, 70 (2002) 223-262. http://dx.doi.org/10.1111/14680262.00274

[24] M. Delattre, V. GENON-CATALOT, A. Samson, Maximum likelihood estimation for stochastic differential equations with random effects, Scandinavian Journal of Statistics, 40 (2013) 322-343. http://dx.doi.org/10.1111/j.1467-9469.2012.00813.x.

[25] R. Liptser, A.N. Shiryaev, Statistics of random Processes: I. general Theory, Springer Science \& Business Media, 2013.

[26] A.W. Van der Vaart, Asymptotic statistics, Cambridge university press, 2000 